\title{
On the globular cluster formation history of NGC 5128
}

\author{
S. Kaviraj ${ }^{1}$, I. Ferreras ${ }^{1,2,3}$, S.-J. Yoon ${ }^{1}$, and S. K. Yi ${ }^{1}$ \\ 1 Department of Physics, University of Oxford, Keble Road, Oxford OX1 3RH, UK \\ e-mail: skaviraj@astro.ox.ac.uk \\ 2 Department of Physics, Institute of Astronomy, ETH Hoenggerberg HPF D8, 8093 Zurich, Switzerland \\ 3 Department of Physics and Astronomy, University College London, Gower Street, London WC1E 6BT, UK
}

Received 20 May 2004 / Accepted 13 January 2005

\begin{abstract}
We deduce the globular cluster formation history of the nearby elliptical galaxy, NGC 5128, by using a chemical enrichment model to accurately reproduce its observed metallicity distribution function (MDF). We derive the observed MDF using recently obtained $U$ and $B$ photometry of the NGC 5128 GC system, with $(U-B)$ used as the metallicity indicator. Our results indicate that the GC system in this galaxy could be the product of two major GC formation episodes. The initial formation episode occured 11-12 Gyr ago creating 65-75 percent of the mass in the GC system. This was followed by a second late formation episode which peaked 2-4 Gyr ago and produced the remaining 25-35 percent of GC mass.
\end{abstract}

Key words. galaxies: elliptical and lenticular, cD - galaxies: evolution - galaxies: formation - galaxies: individual: NGC 5128

\section{Introduction}

Globular cluster (GC) systems have been popular tools in deciphering the star formation histories (SFHs) of galaxies. Their ubiquitous presence in galaxies of all morphological types, coupled with evidence that their creation seems to accompany major star formation episodes (e.g. Larsen \& Richtler 1999) makes them useful tracers of galactic evolution (e.g. Kissler-Patig et al. 1998; van den Bergh 2000; Yoon \& Lee 2002). In addition, GC systems are well represented by simple stellar populations (SSPs) with stars of the same age and chemical composition, which makes them easy to study using SSP models (e.g. Yi et al. 2004).

The exact formation mechanism of GCs has been the subject of much recent debate. Many models for GC formation have been proposed including gaseous mergers (Ashman \& Zepf 1992), in situ formation (e.g. Harris et al. 1995), multiphase collapse (Forbes et al. 1997), dissipationless hierarchical merging (e.g. Côté et al. 1998, 2000, 2002) and hierarchical clustering (Beasley et al. 2003). While none of these models can be conclusively excluded, the widespread discovery of multimodal metallicity distributions in GC populations effectively rules out an extreme version of the monolithic collapse scenario for their formation (see e.g. Forbes et al. 1997). Furthermore, the correlation between the mean metallicity of GCs and galaxy luminosity indicates that chemical enrichment of the GC system is intimately linked to the evolution of the host galaxy (Forbes et al. 1996; Durrell et al. 1996; Forbes \& Forte 2001; Côté et al. 2000; Jordán et al. 2004). An elegant review of the GC formation models mentioned above can be found in West et al. (2004).
The object of this study is NGC 5128, the giant elliptical galaxy in the nearby Centaurus Group (see Israel 1998, for a comprehensive review of NGC 5128). Located at a distance of approximately $3.6 \mathrm{Mpc}$ (e.g. Soria et al. 1996), NGC 5128 is the closest giant elliptical system with an estimated GC population of $1550 \pm 350$ (Harris et al. 1984). It has been widely studied, not only because of its proximity and relative brightness, but also because it displays unusual physical features which suggest that this galaxy is a post-merger remnant. A prominent dust lane containing young stars and HII regions (e.g. Unger et al. 2000; Wild \& Eckart 2000) and a series of optical shells (Malin \& Carter 1983), which have HI (Schiminovich et al. 1994) and molecular CO (Charmandaris et al. 2000) gas associated with them, are considered strong evidence that NGC 5128 underwent a recent merger event within the last $10^{9}$ years. Recently, Rejkuba et al. (2004) suggested that star formation may have stopped as recently as $2 \mathrm{Myr}$ ago in the north-eastern shell of NGC 5128.

In a series of major works, Harris et al. (1999), Harris \& Harris (2000) and Harris \& Harris (2002) performed a comprehensive study of the metallicity distribution of stars in the inner and outer halo of NGC 5128. In this study we deduce the formation history of the GC system of NGC 5128 by accurately reproducing its observed metallicity distribution function (MDF) using a chemical enrichment model. We derive the observed MDF of this elliptical galaxy using recently obtained $U$ and $B$ photometry of 210 clusters in its GC system (Peng et al. 2004a). The integrated $(U-B)$ colour is used as the metallicity indicator because it is sensitive to metallicity via the opacity effect but relatively insensitive to the effective main sequence turn-off temperature $\left(T_{\text {eff }}\right)$ and therefore to age when $T_{\text {eff }} \sim 7000-12000 \mathrm{~K}$ (Yi et al. 2004). Similar 
techniques using $U$ band colours have been used by Rejkuba (2001) and Jordán et al. (2002) who used $(U-V)$ and Hubble Space Telescope WFPC2 (F336W - F547M), respectively. Although these colours are substantially better metallicity indicators than the previously used $(V-I)$ or $(B-V)$, they still change gradually with age and thus are not as effective as $(U-B)$ in determining metallicity (Yi et al. 2004). Our work extends previous studies of NGC 5128 in a number of ways. The large number of confirmed GCs with $U$ band photometry makes it possible to derive statistically significant results. In addition, the relative robustness of $(U-B)$ as a metallicity indicator, compared to other optical colours, makes chemical enrichment an effective approach in modelling the formation history of the NGC 5128 GC system. The use of a consistent chemical enrichment code means that we can effectively transform metallicities into ages.

We begin by briefly checking that, as might be expected from previous theoretical and observational results, a single starburst followed by passive evolution (a monolithic scenario) is incapable of reproducing the observed MDF of the NGC 5128 GCs. Performing this check is not a redundant exercise because our modelling essentially yields relative likelihoods for various models to fit the observed MDF of NGC 5128. It is therefore instructive to compare the quality of fit between monolithic and non-monolithic scenarios. The main thrust of the paper, however, is to explore a double starburst scenario, analysing positions, timescales and relative strengths of the two star formation episodes that best explain the MDF of the NGC 5128 GC system. We provide a coherent picture of the formation history of NGC 5128 GCs based on double starburst scenarios that give excellent fits to the observed MDF and show that our results are consistent with the spectroscopic study of Peng et al. (2004b), who use the agesensitive $\mathrm{H}_{\beta}$ index to age-date the GCs in this dataset.

\section{Derivation of the observed NGC 5128 MDF}

We derive the observed MDF for the NGC 5128 GC system by using photometric data in the $U$ and $B$ bands. We compute the metallicities by overlaying a model $(U-B)$ vs. $(B-V)$ grid on the GC data, with $(U-B)$ used as the metallicity indicator.

The metallicity of each GC is computed by identifying its two bounding iso-metallicity curves, followed by linear interpolation between them. We do not use the same iso-metallicity curves to compute the metallicity error from the $(U-B)$ errors. Instead we treat each error limit as a separate point on the grid and repeat the process described above. Given the irregular spacing and pseudo-horizontal nature of the model iso-metallicity curves (see Fig. 7 in Yi et al. 2004), this results in a more accurate approximation of the metallicity errors for each GC. It should be noted that $(U-B)$ colours (especially redder colours) are affected by the age-metallicity degeneracy. The colour lying in a region of large degeneracy induces a larger metallicity error, since the corresponding metallicity measurement becomes more uncertain. We also note that Yi et al. (2004) did not attempt to accurately derive the ages and metallicities of individual GCs in this dataset. The age and metallicity derivations given in Yi et al. (2004) are rough

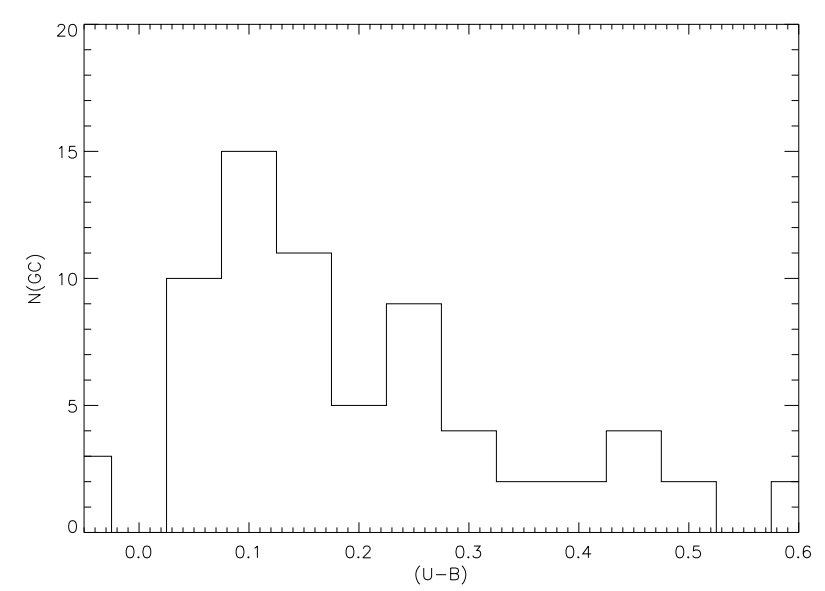

Fig. 1. $(U-B)$ colour distribution derived from final sample of GCs used in this study.

estimates derived from average values of $(B-V)$ and $(U-B)$ in broad bins. The method we use here provides far superior quantification of the metallicity by using a finely interpolated $(U-B)$ grid.

\section{GC selection}

Our photometric data on NGC 5128 contains 210 GCs, some of which suffer from large uncertainties in the $U$ and $B$ magnitudes. In this study we applied a cut of $0.1 \mathrm{mag}$ to the uncertainty in $(U-B)$, retaining 69 GCs out of the original sample of 210 . The reasons for applying a rather stringent magnitude error cut are twofold. Firstly, some GCs have extremely large photometric errors. By using GCs with small error bars we are able to better quantify the metallicity of the GCs that we eventually use along with their associated errors. This in turn results in more constrained estimates for the characteristics of the star formation episodes. Secondly, having performed this magnitude cut we find that the MDF for the entire sample of 210 GCs is very similar to our reduced sample; a KolmogorovSmirnov (KS) test (see e.g. Wall 1996) between the entire sample and the distribution derived after the uncertainty cut yields a KS probability of 42 percent, indicating that the two samples are consistent at the 95 percent confidence level. 15 percent of the final sample of 69 GCs lie within a projected radius of $6^{\prime}$ from the centre of the galaxy (NGC 5128 has an apparent size of $18^{\prime} \times 14^{\prime}$ ) compared to 25 percent of the original sample. 56 percent of the final sample lies within $10^{\prime}$ compared to 59 percent of the original sample. The faint end of the GCLF of the final sample lies at $V=19.7 \mathrm{mag}$, while the faint-magnitude cuts are 20.6 and 20.4 in the $U$ and $B$ bands respectively.

Our aim is to maximise the accuracy of our analysis. The magnitude cut we employ increases the robustness of our final solutions, without transforming the original distribution significantly. Figure 1 presents the $(U-B)$ colour distribution derived from our final sample of clusters and Fig. 2 presents the metallicity distribution derived from the $(U-B)$ colours of our final sample of clusters. We note that our derived MDF is consistent with the NGC $5128 \mathrm{MDF}$ derived using the $\left(C-T_{1}\right)$ index for 205 clusters in Harris et al. (2003). This comparison is shown in the bottom panel of Fig. 2. 


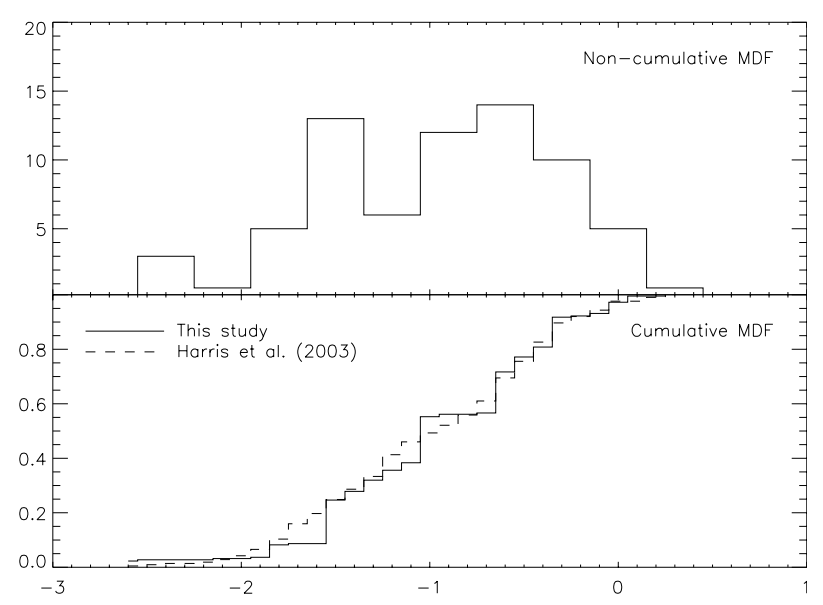

Fig. 2. Top panel: metallicity distribution derived from $(U-B)$ colours of $69 \mathrm{GCs}$ which have $(U-B)$ photometric errors less than 0.1 mag. All photometry is corrected for Galactic extinction. Bottom panel: comparison between the derived metallicity distribution (shown in top panel) and that derived by Harris et al. (2003) using the metallicity sensitive $\left(C-T_{1}\right)$ index.

\section{Star formation and gas infall prescriptions}

Feedback processes from star formation remain poorly understood. Supernovae typically inject large amounts of energy into the inter-stellar medium. The energy deposited can be either kinetic or thermal and the exact description of these processes remains the subject of much debate (see e.g. Katz 1992; Mihos \& Hernquist 1994; Yepes et al. 1997; Ferreras et al. 2002; Silk 2003). Such feedback processes can interrupt or accelerate star formation, so the simple coupling between star formation and the gas density implied by, for example, a Schmidt law (Schmidt 1959) may not hold. In this model, we decouple the star formation rate from the gas density in the system to qualitatively account for such feedback processes from star formation. We take the star formation rate to be a sequence of Gaussian functions independent of the gas density

$\Psi(t)=\sum_{n} \frac{f_{n}}{\sqrt{2 \pi} \sigma_{n}} \exp \frac{-\left(t-\tau_{n}\right)^{2}}{2 \sigma_{n}^{2}}$.

In Eq. (1) $f_{n}$ is the normalisation amplitude, $\sigma_{n}$ the temporal spread and $\tau_{n}$ the centre-point of starburst $n$. With the Gaussian starbursts normalised as shown above, we keep the total area under $\Psi(t)$ and hence the total stellar mass equal to 1 by ensuring that $\sum_{n} f_{n}=1$. We checked that our results do not depend strongly on our choice of mathematical function by repeating our analysis using Lorentzian starbursts, and find that our conclusions remain unchanged.

We assume that the system begins with no gas and use an exponentially decaying gas infall rate

$g_{\text {in }}(t)=\frac{\eta}{\tau_{\text {in }}} \exp \left(\frac{-t}{\tau_{\text {in }}}\right)$

Here $\tau_{\text {in }}$ is the infall timescale, and $\eta$ is a parameter that controls the final gas fraction in the system and is defined below. Noting that,

$\int_{0}^{t_{U}} g_{\text {in }}(t) \mathrm{d} t=\eta\left[1-\exp \left(\frac{-t_{\mathrm{U}}}{\tau_{\text {in }}}\right)\right] \approx \eta$, where $t_{\mathrm{U}}$ is the age of the universe in Gyr, we can force the system to have a final gas fraction $g_{\text {frac }}$ by choosing

$g_{\text {frac }}=\frac{\eta-1}{\eta}$

so that,

$\eta=\frac{1}{1-g_{\mathrm{frac}}}$.

We fix the infall timescale $\tau_{\text {in }}$ to $5 \mathrm{Gyr}$, which leaves the following $3 n$ free parameters in the model for an $n$ starburst scenario

$\left(f_{1}, \ldots, f_{n-2}, \tau_{1}, \ldots, \tau_{n-1}, \sigma_{1}, \ldots, \sigma_{n-1}, g_{\text {frac }}\right)$.

We note that due to the decoupling of the star formation rate from gas density, changing the value of the $\tau_{\text {in }}$ has no impact on our subsequent analysis. Essentially, the behaviour of the system remains unchanged as long as there is a reservoir of gas available to make stars.

Given the prescriptions given above for the star formation and gas infall rates, we compute the metallicity evolution of the GCs following the standard set of chemical enrichment equations as described in Ferreras \& Silk (2000). We use the yields of Thielemann et al. (1996) for stellar masses $M_{\star}>10 M_{\odot}$ and van den Hoek \& Groenewegen (1997) for lower mass stars. For a given set of parameters we integrate the equations to allow us to compute the MDF, that is then compared with the observed $\mathrm{MDF}$ via a KS test.

\section{Derivation of best-fit parameters}

We begin our analysis by comparing a single starburst scenario, which describes a monolithically evolving system, to the more realistic ones that underpin this study. We then refine our analysis by adding another starburst and perform a detailed study of the resulting six dimensional parameter space. We also check that small perturbations to the two major star formation episodes do not affect the best-fit values for our parameters. This sequence is repeated for four final gas fractions $g_{\text {frac }}=0.1,0.2,0.35,0.5$. Since we are modelling an elliptical galaxy, the value of $g_{\text {frac }}$ is likely to be low, probably somewhere in the region $0.1-0.35$. In a recent work, Sanderson et al. (2003) finds the (hot) gas fraction in ellipticals within $R_{200}$ to be approximately 0.1 .

We compare the MDF predicted by our model to the observed GC MDF using the KS test. The best-fit values of parameters are calculated by marginalising their KS probabilities, which involves summing (integrating) out the probability dependence of all other parameters, leaving a probability distribution for each parameter that is independent of all others in the model. For example, in a double starburst scenario, the marginalised probability distribution for the parameter $\tau_{1}$ is calculated as

$P\left(\tau_{1}\right)=\varsigma \sum_{i, j, k} P\left(\tau_{1}, \tau_{2 i}, \sigma_{1 j}, \sigma_{2 k}\right)$

where $\varsigma$ is a normalising constant which ensures that $P\left(\tau_{1}\right)$ integrates to 1 . This probability distribution will not, in general, 
be symmetric and therefore should not be approximated by a Gaussian.

We take the best-fit value of a parameter as the value at which its marginalised probability function peaks. In addition, we define errors for these parameters superficially similar to the one-sigma errors frequently used for Gaussian probability distributions. If $X$ is the most likely value of a parameter $x$, then we define the positive error $x_{+}$as

$\int_{X}^{x_{+}} P(x) \mathrm{d} x=0.34 \int_{X}^{\infty} P(x) \mathrm{d} x$,

and similarly the negative error, $x_{-}$, as

$$
\int_{x_{-}}^{X} P(x) \mathrm{d} x=0.34 \int_{-\infty}^{X} P(x) \mathrm{d} x .
$$

Finally, given the fact that each GC has a metallicity error associated with it, we check that the positions of the most likely values for our model parameters are robust with respect to these errors. We perform this check by adding random noise to our observed MDF and producing KS values for these noisy MDFs. The noise is added by running through each GC and changing its metallicity to one of its error points randomly. This process is repeated a hundred times to generate a hundred noisy MDFs. We have checked that the KS values do not deviate significantly from the fiducial value and find that the KS values are suitably robust (within 6 percent of the original values) when the fiducial MDF is perturbed in this way. This is mainly a result of choosing GCs with small photometric errors.

\section{Results and analysis}

\subsection{Double starburst scenarios}

For a single starburst scenario, the maximum KS probability we achieve in our model is around 2 percent, significantly lower than the double starburst scenarios we explore later. The most favoured values of $\tau$ and $\sigma$ for the single starburst scenario are $1 \mathrm{Gyr}$ and 1.35 respectively. A single starburst model fails to reproduce, with any acceptable accuracy, the observed MDF of NGC 5128.

With two starbursts we obtain a six dimensional parameter space with the star formation rate as the sum of two Gaussians

$$
\Psi(t)=\frac{f_{1}}{\sqrt{2 \pi} \sigma_{1}} \exp \frac{-\left(t-\tau_{1}\right)^{2}}{2 \sigma_{1}^{2}}+\frac{1-f_{1}}{\sqrt{2 \pi} \sigma_{2}} \exp \frac{-\left(t-\tau_{2}\right)^{2}}{2 \sigma_{2}^{2}} .
$$

Table 1 shows the best-fit values for the starburst parameters $\left(f_{1}, f_{2}, \tau_{1}, \tau_{2}, \sigma_{1}, \sigma_{2}\right)$ for final gas fractions of 0.5 to 0.1 . Note that in Table 1, Subscript " 1 " indicates the initial (early) starburst and Subscript 2 indicates the second (late) starburst.

In Fig. 3, we present the best KS values produced in the double starburst models. The single starburst case is shown, for comparison, by the solid line at 2 percent. In Fig. 4 we present three GC formation histories which yield the highest KS probabilities (see shaded region in Fig. 3) by showing the formation histories for favoured values $\left(f_{1}, f_{2}\right)$ in a system with a final gas fraction of 0.35 .

Figure 3 suggests that an intermediate gas fraction of 0.35 with $\left(f_{1}, f_{2}\right)=(0.35,0.65)$ is the most favoured model, so that

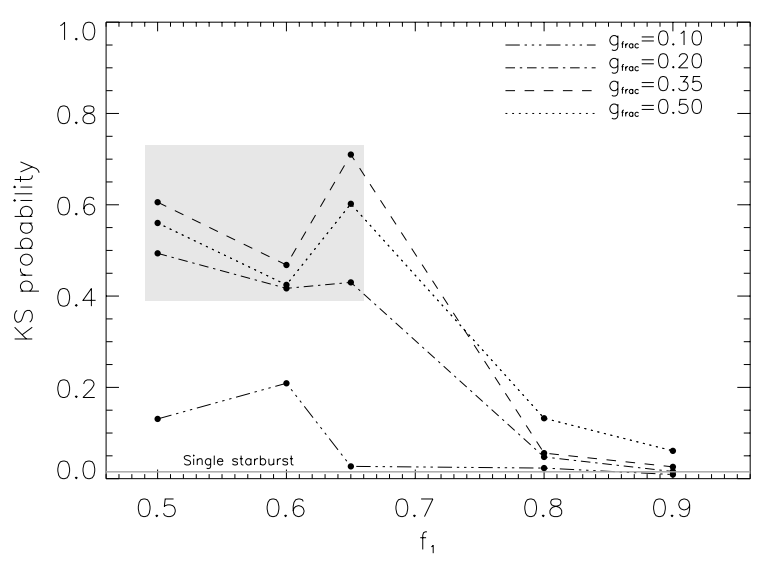

Fig. 3. Best KS probabilities for various values of $f_{1}$ and for four final gas fractions $g_{\text {frac }}$. The shaded region shows a set of degenerate models that have high $\mathrm{KS}$ probabilities and therefore cannot be discounted completely. The KS probability for a single starburst scenario is shown by the solid line at 2 percent.

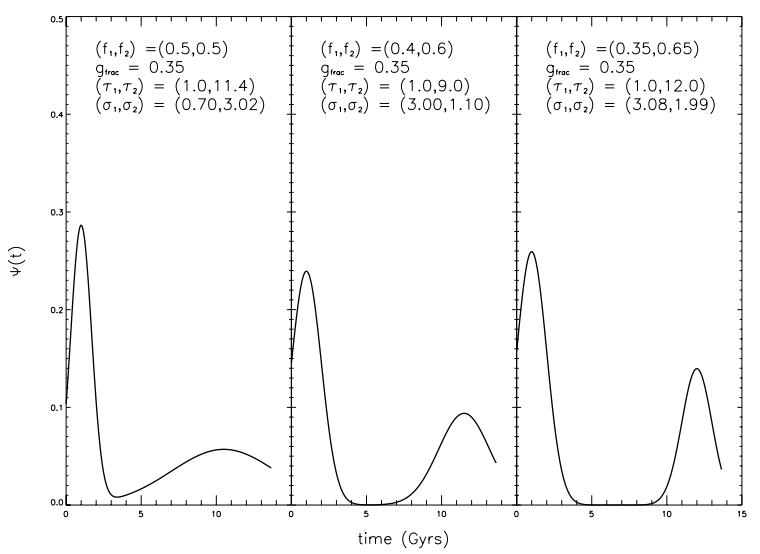

Fig. 4. Three GC formation histories corresponding to the best KS probabilities shown in the shaded region in Fig. 3.

roughly one-third of the mass in the GC system is predicted to have been formed in a late starburst centred around $2 \mathrm{Gyr}$ ago. Figure 5 shows the mass fractions formed at various ages and metallicities in this model, and Fig. 6 compares the MDF predicted by this model to the observed MDF.

Although the favoured model provides the best-fit, the shaded region in Fig. 3 shows a set of degenerate solutions consisting of models producing high KS values, all of which reasonably reproduce the observed NGC $5128 \mathrm{MDF}$ and therefore cannot be completely discounted. Changing the relative sizes of the starbursts even further results in the KS probability declining rapidly. This is clearly expected, since in the limit $f_{1} \rightarrow 0$, we recover a single starburst, already established as being incapable of reproducing the observed NGC 5128 MDF. While the position of the first starburst is fairly robust in the best-fit models, the second starburst tends to vary, so that we are left with a dispersion of $\sim 2$ Gyr in the second starburst's position. This reflects the fact that uncertainties in colours and in the age-metallicity degeneracy (discussed in Sect. 2) induce uncertainties in the metallicity derivations for our GCs, which in turn limits our resolution of the position of the late GC formation episode. Averaging over all the best-fit models (shown 
Table 1. Best-fit values and errors for the double starburst scenarios with final gas fractions in the range 0.1-0.5. Note that Subscript "1" indicates the initial (early) starburst and Subscript 2 the second (late) one.

\begin{tabular}{|c|c|c|c|c|c|}
\hline \multicolumn{6}{|c|}{ Final gas fraction $=0.5$} \\
\hline$\left(f_{1}, f_{2}\right)$ & $(0.5,0.5)$ & $(0.6,0.4)$ & $(0.65,0.35)$ & $(0.8,0.2)$ & $(0.9,0.1)$ \\
\hline$\tau_{1}$ & $0.72_{-0.31}^{+0.12}$ & $1.00_{-0.14}^{+0.09}$ & $0.51_{-0.02}^{+0.18}$ & $1.01_{-0.04}^{+0.13}$ & $1.00_{-0.21}^{+0.14}$ \\
\hline$\tau_{2}$ & $5.00_{-0.26}^{+0.22}$ & $9.99_{-0.26}^{+0.46}$ & $10.00_{-1.00}^{+0.44}$ & $11.90_{-0.96}^{+0.13}$ & $11.10_{-0.95}^{+0.25}$ \\
\hline$\sigma_{1}$ & $0.70_{-0.12}^{+0.01}$ & $0.70_{-0.03}^{+0.15}$ & $1.00_{-0.05}^{+0.12}$ & $1.02_{-0.08}^{+0.23}$ & $2.00_{-0.09}^{+0.13}$ \\
\hline$\sigma_{2}$ & $3.55_{-0.27}^{+0.21}$ & $3.58_{-0.17}^{+0.24}$ & $1.99_{-0.25}^{+0.80}$ & $0.60_{-0.02}^{+1.02}$ & $1.49_{-0.23}^{+0.17}$ \\
\hline KS prob & 0.57 & 0.43 & 0.61 & 0.13 & 0.06 \\
\hline \multicolumn{6}{|c|}{ Final gas fraction $=0.35$} \\
\hline$\left(f_{1}, f_{2}\right)$ & $(0.5,0.5)$ & $(0.6,0.4)$ & $(0.65,0.35)$ & $(0.8,0.2)$ & $(0.9,0.1)$ \\
\hline$\tau_{1}$ & $1.00_{-0.09}^{+0.20}$ & $1.00_{-0.09}^{+0.20}$ & $1.00_{-0.06}^{+0.20}$ & $1.00_{-0.06}^{+0.23}$ & $1.00_{-0.09}^{+0.20}$ \\
\hline$\tau_{2}$ & $11.40_{-0.54}^{+0.07}$ & $9.00_{-0.93}^{+0.07}$ & $12.00_{-0.93}^{+0.07}$ & $9.00_{-0.88}^{+0.83}$ & $12.10_{-0.92}^{+0.62}$ \\
\hline$\sigma_{1}$ & $3.02_{-0.93}^{+0.38}$ & $1.10_{-0.09}^{+0.20}$ & $1.13_{-0.09}^{+0.19}$ & $1.99_{-0.22}^{+0.31}$ & $2.99_{-0.42}^{+0.28}$ \\
\hline$\sigma_{2}$ & $0.70_{-0.04}^{+0.16}$ & $3.00_{-0.28}^{+0.36}$ & $0.99_{-0.15}^{+0.48}$ & $3.08_{-0.55}^{+0.36}$ & $3.05_{-0.73}^{+0.36}$ \\
\hline KS prob & 0.61 & 0.47 & 0.73 & 0.05 & 0.03 \\
\hline \multicolumn{6}{|c|}{ Final gas fraction $=0.2$} \\
\hline$\left(f_{1}, f_{2}\right)$ & $(0.5,0.5)$ & $(0.6,0.4)$ & $(0.65,0.35)$ & $(0.8,0.2)$ & $(0.9,0.1)$ \\
\hline$\tau_{1}$ & $0.99_{-0.04}^{+0.15}$ & $1.00_{-0.06}^{+0.11}$ & $1.01_{-0.04}^{+0.13}$ & $0.99_{-0.04}^{+0.15}$ & $1.00_{-0.04}^{+0.16}$ \\
\hline$\tau_{2}$ & $10.50_{-0.88}^{+0.33}$ & $11.45_{-0.48}^{+0.19}$ & $11.20_{-0.61}^{+0.15}$ & $10.90_{-1.32}^{+0.24}$ & $10.70_{-1.46}^{+0.24}$ \\
\hline$\sigma_{1}$ & $0.76_{-0.05}^{+0.19}$ & $0.99_{-0.06}^{+0.11}$ & $1.00_{-0.07}^{+0.14}$ & $2.00_{-0.15}^{+0.19}$ & $2.99_{-0.61}^{+0.14}$ \\
\hline$\sigma_{2}$ & $3.50_{-0.18}^{+0.23}$ & $1.74_{-0.27}^{+0.31}$ & $1.00_{-0.10}^{+0.37}$ & $2.00_{-0.22}^{+0.38}$ & $2.50_{-0.20}^{+0.66}$ \\
\hline KS prob & 0.49 & 0.43 & 0.44 & 0.04 & 0.02 \\
\hline \multicolumn{6}{|c|}{ Final gas fraction $=0.1$} \\
\hline$\left(f_{1}, f_{2}\right)$ & $(0.5,0.5)$ & $(0.6,0.4)$ & $(0.65,0.35)$ & $(0.8,0.2)$ & $(0.9,0.1)$ \\
\hline$\tau_{1}$ & $1.12_{-0.12}^{+0.11}$ & $0.91_{-0.67}^{+0.12}$ & $1.45_{-0.12}^{+0.20}$ & $1.20_{-0.12}^{+0.18}$ & $1.00_{-0.34}^{+0.18}$ \\
\hline$\tau_{2}$ & $11.20_{-0.78}^{+0.15}$ & $12.06_{-0.28}^{+0.15}$ & $11.40_{-0.87}^{+0.11}$ & $12.00_{-0.78}^{+0.56}$ & $10.89_{-0.80}^{+0.14}$ \\
\hline$\sigma_{1}$ & $0.72_{-0.18}^{+0.29}$ & $0.99_{-0.03}^{+0.12}$ & $1.00_{-0.05}^{+0.11}$ & $3.00_{-0.06}^{+0.29}$ & $2.50_{-0.35}^{+0.10}$ \\
\hline$\sigma_{2}$ & $3.53_{-0.58}^{+0.28}$ & $3.46_{-0.17}^{+0.23}$ & $2.00_{-0.30}^{+0.17}$ & $1.00_{-0.12}^{+0.36}$ & $3.00_{-0.18}^{+0.56}$ \\
\hline KS prob & 0.14 & 0.22 & 0.03 & 0.03 & 0.02 \\
\hline
\end{tabular}

in the shaded region in Fig. 3) and taking the one-sigma errors into account for the parameters given in Table 1, our results therefore indicate that the observed MDF of the NGC 5128 GCs can be reproduced accurately by a formation scenario where roughly 65-75 percent of the mass in the GC system was formed in an initial starburst 11-12 Gyr ago, followed by a second late starburst which peaked 2-4 Gyr ago and produced the remaining 25-35 percent of GC mass.

\subsection{Perturbations to the double starburst case}

As mentioned above, the $n$ starburst scenario contains $3 n$ free parameters. Clearly, as we make more free parameters available to the model, we should expect to do better in terms of matching the observed NGC 5128 MDF. We note first that the KS values from the double starburst scenarios are significantly large. Refining the model further would not make an appreciable 


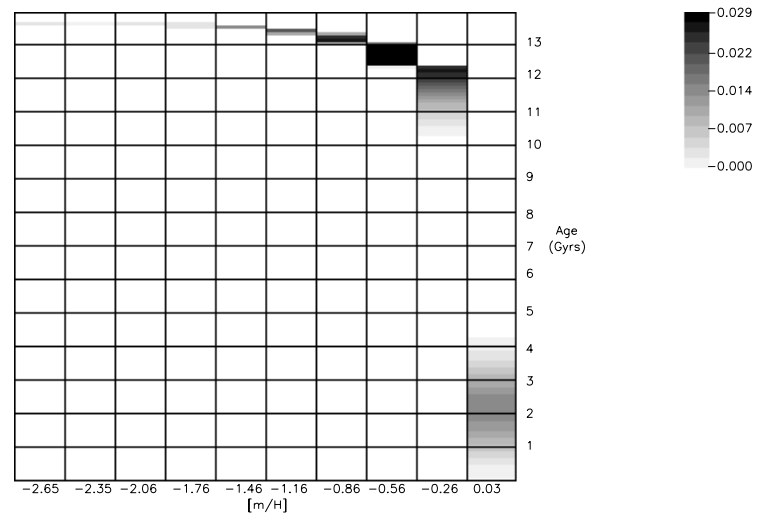

Fig. 5. Age-metallicity grid showing the fraction of stellar mass predicted by the favoured model as a function of age and metallicity. The key indicates stellar fractions corresponding to the levels of shading in the plot.

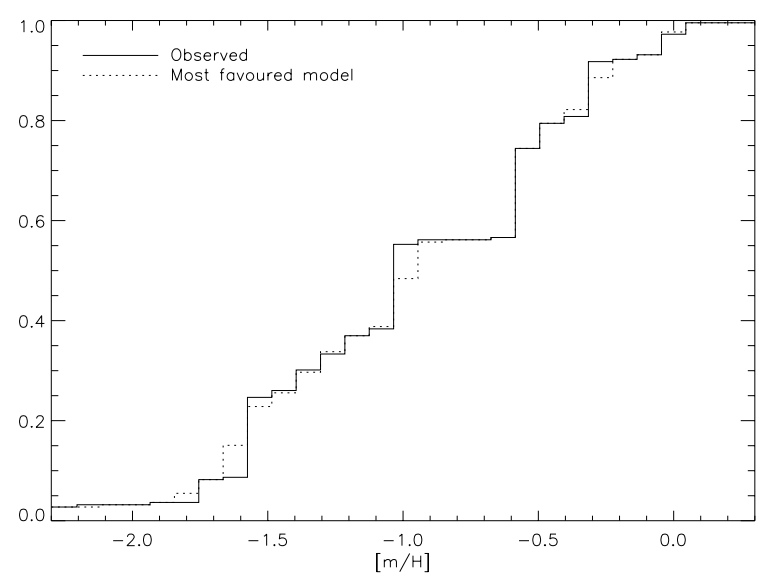

Fig. 6. Comparison between observed MDF and that predicted by the favoured model (which gives the highest KS probabillity).

difference in the KS probabilities, certainly not as much as in the transition from the single to the double starburst case. In addition, given the errors in the observed MDF and the resolution of our model, further refinement would not provide much greater insight into the GC formation history of this galaxy.

Therefore, instead of repeating the study above with a wholesale three starburst analysis, we chose to treat the third starburst as a perturbation on the double starburst system. We were interested in studying how the KS probabilities change if we apply a small perturbation to each of the major star formation episodes. This simple perturbation analysis gave an indication of how localised these double starburst KS hotspots were in the nine parameter triple starburst space.

Figure 7 shows the best KS values achieved after applying a 5 percent pertubation i.e. $f_{\text {perturb }}=0.05$ as a fraction of the best KS values in the corresponding unperturbed double starburst scenario. In almost all cases the KS value improves only marginally. We conclude from this analysis that the KS hotspot is not extremely localised in the nine parameter triple starburst space and does appear to be stable at least under perturbations of sizes 5 percent and below. Although we have sampled only a small fraction of this nine-dimensional parameter space,

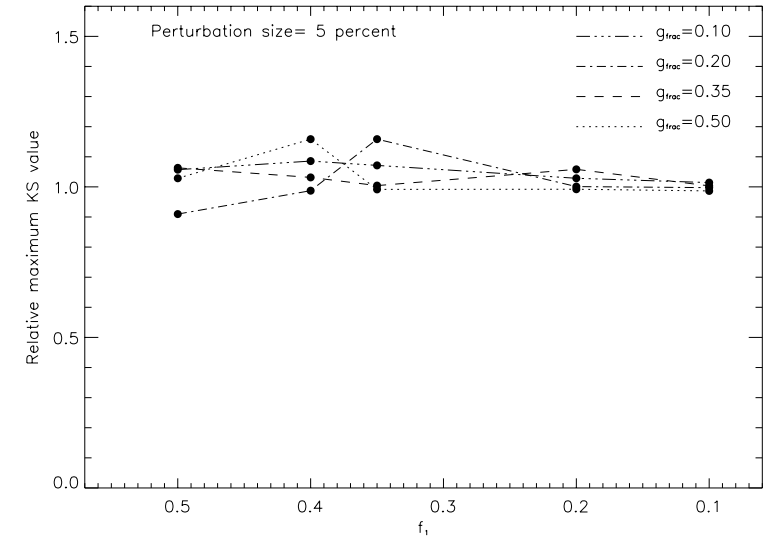

Fig. 7. KS values for perturbed double starburst scenarios as a fraction of the KS values in the unperturbed case. The size of the perturbation applied to the system is 5 percent.

the resistance to small perturbations indicates that the double burst scenarios are reasonably stable and robust solutions.

\section{Discussion and conclusions}

We studied the formation history of the NGC 5128 GC system by using a chemical enrichment model to accurately reproduce its observed MDF derived from recently obtained $U$ and $B$ photometry. Our results show that the GC system in this galaxy could be the product of two major GC formation episodes, although we do not have adequate resolution in the model or in the observed MDF to make any reliable claim about the possibility of additional formation events.

The double starburst analysis produces high KS probabilities and therefore good fits to the observed MDF, with best-fit models (shaded region in Fig. 3) that favour an initial GC formation episode 11-12 Gyr ago which produced 65-75 percent of mass in the GC system, with a second late formation episode approximately 2-4 Gyr ago producing the remaining 25-35 percent. The late starburst results in a small fraction ( $\sim 5$ percent) of the stellar mass in the GC system potentially having ages less than 1 Gyr. If NGC 5128 has over 1500 clusters, then we might expect a handful of those clusters to have ages less than $1 \mathrm{Gyr}$. We have not made any a priori assumptions about the driving mechanisms behind star formation episodes in our model. While a single starburst followed by passive evolution can clearly be discounted, the nature of our model admits any scenario where multiple episodes of GC formation are possible.

Our results are in general agreement with Beasley et al. (2003), who obtain good fits to the observed NGC 5128 MDF using a semi-analytical galaxy formation model with metalpoor GC formation halted at $z \sim 5$. A quantitative similarity between this study and that of Beasley et al. (2003) is that the initial GC formation episode (first Gaussian starburst) in our model, which gave rise to the metal-poor GC subpopulation, decays rapidly to virtually zero (see Fig. 4) within $\sim 2$ Gyr of the formation of the galaxy; i.e. there is effective truncation of metal-poor GC production at high redshift $(z \sim 3-4)$. This closely resembles the truncation employed by 
Beasley et al. (2003), and the similarity is probably due to the fact that our model is comparable to the chemical enrichment prescription employed in the semi-analytical model of Beasley et al. (2003). Since the object of both studies is to reproduce metallicity distributions this resemblance is not unexpected.

This study would not be complete without a comparison of our results to those of Peng et al. (2004b), who performed a spectroscopic analysis of the GC dataset used in this paper. Based on the age-sensitive $\mathrm{H}_{\beta}$ index, they conclude that metal-poor GCs in NGC 5128 have ages comparable to those in the Milky Way, i.e. 12 Gyr (e.g. Krauss \& Chaboyer 2003), and that the metal-rich GCs are consistent with a mean age of $5_{-2}^{+3}$ Gyr. We thus find that our age estimates, derived using a chemical enrichment approach to exploit metallicity-sensitive photometric colours, are consistent with a study of the same objects using age-sensitive spectroscopic indices.

Our study demonstrates the potential of a chemical enrichment approach in deciphering the formation histories of the GC system in galaxies. As more age and metallicity-sensitive spectro-photometric data become available, methods such as the one used in this study will enable us to set robust constraints on the way galaxies either form or incorporate GCs, crucial to our understanding of galaxy formation.

Acknowledgements. We are grateful to the referee for numerous comments and suggestions that improved the quality of this manuscript. We are indebted to Eric Peng for providing the NGC 5128 data on which this work is based, prior to publication. We warmly thank Andrés Jordán for his very careful reading of this manuscript and numerous useful comments. We also thank Roger Davies, Joseph Silk and Julien Devriendt for constructive remarks regarding this study. S.K. acknowledges PPARC grant PPA/S/S/2002/03532. This research was supported by PPARC Theoretical Cosmology Rolling Grant PPA/G/O/2001/00016 (S. K. Yi and I. Ferreras), the Glasstone Fellowship and the Post-doctoral Fellowship Program of Korea Science \& Engineering Foundation (S.-J. Yoon) and made use of Starlink computing facilities at the University of Oxford.

\section{References}

Ashman, K. M., \& Zepf, S. E. 1992, ApJ, 384, 50

Beasley, M. A., Harris, W. E., Harris, G. L. H., \& Forbes, D. A. 2003, MNRAS, 340, 341

Côté, P., Marzke, R. O., \& West, M. J. 1998, ApJ, 501, 554

Côté, P., Marzke, R. O., West, M. J., \& Minniti, D. 2000, ApJ, 533, 869

Côté, P., West, M. J., \& Marzke, R. O. 2002, ApJ, 567, 853

Charmandaris, V., Combes, F., \& van der Hulst, J. M. 2000, A\&A, 356, L1

Durrell, P. R., Harris, W. E., Geisler, D., \& Pudritz, R. E. 1996, AJ, 112,972
Ferreras, I., \& Silk, J. 2000, ApJ, 532, 193

Ferreras, I., Scannapieco, E., \& Silk, J. 2002, ApJ, 579, 247

Forbes, D. A., \& Forte, J. C. 2001, MNRAS, 322, 257

Forbes, D. A., Franx, M., Illingworth, G. D., \& Carollo, C. M. 1996, ApJ, 467, 126

Forbes, D. A., Brodie, J. P., \& Grillmair, C. J. 1997, AJ, 113, 1652

Harris, G. L. H., \& Harris, W. E. 2000, AJ, 120, 2423

Harris, G. L. H., Hesser, J. E., Harris, H. C., \& Curry, P. J. 1984, ApJ, 287,175

Harris, G. L. H., Harris, W. E., \& Poole, G. B. 1999, AJ, 117, 855

Harris, G. L. H., Geisler, D., Harris, W. E., et al. 2003, in Extragalactic Globular Cluster Systems, Proceedings of the ESO Workshop held in Garching, Germany, 27-30 August 2002, 113

Harris, W. E., \& Harris, G. L. H. 2002, AJ, 123, 3108

Harris, W. E., Pritchet, C. J., \& McClure, R. D. 1995, ApJ, 441, 120

Israel, F. P. 1998, A\&AR, 8, 237

Jordán, A., Côté, P., West, M. J., \& Marzke, R. O. 2002, ApJ, 576, L113

Jordán, A., Côté, P., West, M. J., et al. 2004, AJ, 127, 24

Katz, N. 1992, ApJ, 391, 502

Kissler-Patig, M., Forbes, D. A., \& Minniti, D. 1998, MNRAS, 298, 1123

Krauss, L. M., \& Chaboyer, B. 2003, Science, 299, 65

Larsen, S. S., \& Richtler, T. 1999, A\&A, 345, 59

Malin, D. F., \& Carter, D. 1983, ApJ, 274, 534

Mihos, J. C., \& Hernquist, L. 1994, ApJ, 437, 611

Peng, E. W., Ford, H. C., \& Freeman, K. C. 2004a, ApJS, 150, 367

Peng, E. W., Ford, H. C., \& Freeman, K. C. 2004b, ApJ, 602, 705

Rejkuba, M. 2001, A\&A, 369, 812

Rejkuba, M., Greggio, L., \& Zoccali, M. 2004, A\&A, 415, 915

Sanderson, A. J. R., Ponman, T. J., Finoguenov, A., Lloyd-Davies, E. J., \& Markevitch, M. 2003, MNRAS, 340, 989

Schiminovich, D., van Gorkom, J. H., van der Hulst, J. M., \& Kasow, S. 1994, ApJ, 423, L101

Schmidt, M. 1959, ApJ, 129, 243

Silk, J. 2003, MNRAS, 343, 249

Soria, R., Mould, J. R., Watson, A. M., et al. 1996, ApJ, 465, 79

Thielemann, F., Nomoto, K., \& Hashimoto, M. 1996, ApJ, 460, 408

Unger, S. J., Clegg, P. E., Stacey, G. J., et al. 2000, A\&A, 355, 885

van den Bergh, S. 2000, PASP, 112, 932

van den Hoek, L. B., \& Groenewegen, M. A. T. 1997, A\&AS, 123, 305

Wall, J. V. 1996, QJRAS, 37, 519

West, M. J., Côté, P., Marzke, R. O., \& Jordán, A. 2004, Nature, 427, 31

Wild, W., \& Eckart, A. 2000, A\&A, 359, 483

Yepes, G., Kates, R., Khokhlov, A., \& Klypin, A. 1997, MNRAS, 284, 235

Yi, S. K., Peng, E., Ford, H., Kaviraj, S., \& Yoon, S. J. 2004, MNRAS, 349, 1493

Yoon, S. J., \& Lee, Y. 2002, Science, 297, 578 